\title{
Mobile apps for HIV prevention: how do they contribute to our epidemic response for adolescents and young adults?
}

\author{
Patrick S. Sullivan ${ }^{1 \wedge}$, Lisa Hightow-Weidman ${ }^{2}$ \\ ${ }^{1}$ Department of Epidemiology, Rollins School of Public Health, Emory University, Atlanta, GA 30329, USA; ${ }^{2}$ Institute for Global Health and \\ Infectious Diseases, University of North Carolina, Chapel Hill, NC, USA \\ Contributions: (I) Conception and design: All authors; (II) Administrative support: None; (III) Provision of study materials or patients: None; (IV) \\ Collection and assembly of data: None; (V) Data analysis and interpretation: None; (VI) Manuscript writing: All authors; (VII) Final approval of \\ manuscript: All authors. \\ Correspondence to: Patrick S. Sullivan, DVM, PhD. Department of Epidemiology, Rollins School of Public Health, Emory University, 1518 Clifton \\ Road NE, Atlanta, GA 30329, USA. Email: pssulli@emory.edu.
}

\begin{abstract}
Mobile health (mHealth) tools to address the HIV epidemic have proliferated in recent years. Yet when applied to the United States (US) epidemic, which is driven by new HIV infections among men who have sex with men (MSM), it is not clear how mHealth tools fit in the overall portfolio of biobehavioral prevention interventions and clinical services proven to be efficacious. Adolescent and young adult MSM are particularly vulnerable and reducing HIV incidence among this priority population will require substantial levels of uptake of multiple prevention strategies (i.e., HIV testing, condom use, sexually transmitted infection (STI) testing, pre-exposure prophylaxis (PrEP), and treatment for those with HIV infection). Starting from the premise that adolescents are avid consumers of technology, this paper considers the particular strengths and opportunities of mHealth tools to address HIV prevention and provides examples of mHealth approaches that have been tested or are in development in these areas. Even after mHealth interventions are proven effective, there will be important intervening steps before such tools can be deployed and integrated into existing prevention programs given the diverse landscape of prevention service delivery. We anticipate some of the likely barriers to broad implementation of proven mHealth interventions in the context of the US public health funding and service delivery infrastructure and provide recommendations to increase efforts for future scale-up and dissemination.
\end{abstract}

Keywords: HIV prevention; mHealth tools; public health services; pre-exposure prophylaxis (PrEP)

Received: 21 February 2020; Accepted: 05 June 2020; Published: 20 April 2021.

doi: $10.21037 /$ mhealth-20-71

View this article at: http://dx.doi.org/10.21037/mhealth-20-71

\section{Background}

Mobile health (mHealth) and other digital health interventions (DHIs) have proliferated in recent years. A 2015 review of mobile applications (apps) related to HIV prevention revealed that most apps $(71 \%)$ were developed by a nonacademic or public health institution, were free $(83 \%)$, and reported information about one or more HIV prevention modalities (53\%). However, only $7 \%$ of the apps focused specifically on men who have sex with men (MSM), and none dealt with post-exposure prophylaxis (PEP) (1). Since that time, updated inventories of mHealth interventions have been produced $(2,3)$, mobile apps for HIV prevention have proliferated, and several apps have been tested in pilot studies or randomized trials that incorporate biomedical prevention options, including PrEP information and referrals, in their content (4-7). An updated

^ORCID: 0000-0002-7728-0587. 
inventory of mHealth tools for HIV prevention from the published scientific literature demonstrated that most HIV prevention mHealth tools for HIV-negative MSM focus on education and testing, rather than service delivery or linkage to care (3). Among 84 interventions included in this review, $31(37 \%)$ focused specifically on adolescent and young adult MSM, highlighting the expansion of research using digital modalities in this group. What is lacking, however, is clarity regarding how DHIs fit into an overall strategy to address the HIV epidemic among adolescent and young adult MSM by increasing the uptake of prevention services, including HIV testing and PrEP.

In the paper, we consider several key questions that will situate mHealth tools in the epidemic response, focusing on relevance to HIV prevention for MSM and for adolescent and young adult MSM. We will illustrate the specialized functions for digital tools that have been developed and/or tested for efficacy within specific interventions, highlighting how these functions may be particularly relevant or impact for youth. Finally, we will consider how mHealth tools that are proven efficacious can be integrated into the broader public health response to the HIV epidemic among young MSM (YMSM) in the United States (US).

\section{What opportunities for engaging YMSM in HIV prevention are offered by mHealth?}

mHealth tools offer both reach and functions that are highly relevant to HIV prevention. In terms of reach, mHealth tools offer opportunities to provide specialized services [e.g., lesbian, gay, bisexual, transgender (LGBT)-friendly counseling or referrals (8), access to PrEP (9-11), prevention messages (5), and prevention commodities (1)] to MSM who cannot access them due to proximity (12), stigma, or concerns about confidentiality (13). This is especially true for MSM who live in rural areas, who are less likely to receive routinely recommended HIV prevention services, such as HIV testing, sexually transmitted infection (STI) testing, or PrEP services (14). It is important to note that, although DHIs offer opportunities to reach rural MSM, few DHIs have been developed that are specifically adapted to this important group (15). Reach also relates to the ability to provide services to men in disparate geographic areas: DHIs have been conducted that have enrolled MSM in the US nationally, demonstrating feasibility of providing interventions to a national service area from a single service location (16-19).

\section{Specialized functions}

\section{Providing rich media}

Because of increases in data speed in the US (20), it is feasible to deliver rich media, including videos or animations such as graphics interchange format (GIFs). Delivering information through videos and animations can help make for a more engaging user experience, and may increase comprehension of key information (21). MSM who tested pilot versions of a comprehensive HIV prevention app, MyChoices, originally developed for adult MSM, suggested decreasing text-heavy presentations of HIV prevention information in favor of GIFs or videos for presentation of content (6). Videos and images may also address literacy concerns that may limit youth's comprehension of content. A study among MSM (median age 26 years) found that the use of video-delivered informed consent in health research increased comprehension of the elements of the informed consent process (22). Exploiting youth appetites for video content should be prioritized within future DHIs.

\section{Tailoring interventions}

Digital health tools are uniquely suited to tailoring intervention content and information based on user characteristics and preferences. Tailoring can be done based on specific prevention needs [e.g., providing information for antiretroviral (ART) treatment for users with HIV, and for PrEP or other prevention tools for users who are HIVnegative], location (e.g., using geolocation to recommend HIV testing or PrEP clinics that are nearby), or based on personal values or preferences [e.g., framing health recommendations in terms of the values of users $(23,24)]$. Further, mHealth tools can account for the fact that the needs and preferences of users change over time. This can be particularly relevant for YMSM who, in the course of development, experience cognitive, physical and emotional changes that may manifest in fluctuating periods of risk or rapid partner turnover. For example, an evaluation of the M-Cubed mobile app reassessed the levels of HIV risk after three months of use, and different prevention messages were provided to users based on their current levels of risk for HIV infection (5). Tailoring can also relate to the frequency of recommended services; for example, users of the HealthMindr app are offered a quiz to determine an optimal schedule for HIV testing based on their risks for HIV (25), and the Seattle/King County Public Health 
Department offered a similar digital tool ("Find your frequency") to support MSM to make a plan for episodic HIV testing (26).

\section{Providing reminders}

mHealth apps can provide reminders for routine health services [e.g., HIV testing every 6 months; $(6,25)]$ or to support pill taking [for example for PrEP or ART; (27)]. A meta-analysis of text-based reminders for medication adherence showed a significant benefit for chronic disease conditions (28) and some reminder systems improved ART adherence (29); two-way messaging proving more efficacious than one-way messages (30). Sustained efficacy of routine text message reminders for youth has proved limited and there is a need to make reminders more personalized and contextually relevant. Within P3, a PrEP adherence app for YMSM, reminders are tied to activities youth perform on a daily and consistent basis (e.g., brushing their teeth, plugging in their phone at night, opening Instagram), thereby facilitating habit building around PrEP medication taking. Consideration of exploiting the inherent capabilities of smartphones such as geolocation can also allow for reminders to be not only time but location dependent. Reminders that youth perceive as untimely (e.g., in presence of others who don't know they use $\mathrm{PrEP}$ or due to a schedule change may not have access to their medications) can be adjusted to ensure that they are received when pills are present (e.g., they arrive home) rather than simply dismissing the reminder.

\section{Locate services}

Service locators can promote use of key HIV prevention services by helping users identify the right type of service that is in close proximity. The distance to a service location for HIV services matters: in Atlanta, MSM who had longer commute times to their regular care appointments were more likely to have missed care appointments than were men who received care closer to their homes (31). This may be particularly important for younger MSM who may lack personal vehicles and have to rely on others for rides or utilize public transportation. For HIV prevention and care, locators can provide geolocation-informed referrals to HIV testing, STI testing, HIV care, and PrEP services (32). If additional information about service facilities, such as forms of insurance accepted, services offered in multiple languages, times of operation, or whether the facility near a bus stop are available, faceted searches can be offered to help tailor service locations. Such faceted location searches can also incorporate data on service features collected specifically for the app users. For example, Get Connected (24), an mHealth intervention for MSM, utilizes a process of "secret shoppers" to curate lists of HIV testing locations known to be LGBT-affirming and to provide an adequate standard of care to LGBT youth (33). Intervention apps that utilize geofencing, a location-based service in which the app uses GPS, Wi-Fi or cellular data to trigger a preprogrammed action when a mobile device enters or exits a virtual boundary set up around a geographical location, to trigger HIV testing reminders when youth are near testing sites, are being explored within the LYNX and MyChoices apps. This strategy may be particularly impactful among youth who are less likely to engage in planning behaviors.

\section{Screening (and rescreening) for appropriate services}

In HIV prevention, some services have specific eligibility criteria. For example, PrEP is indicated if specific behavioral criteria or a history of STIs is present (34), and PEP is indicated after sex based on the time frame after exposure, which bodily fluids were contacted, and the perceived HIV serostatus of the sex partner (35). These decisions processes can be readily translated within DHIs into short "quizzes" or interactive activities that YMSM can take either periodically (to assess PrEP eligibility), or on-demand (after a sexual exposure if there is a concern that PEP might be indicated). Gamifying these assessments, by rewarding users with badges or rewards based on completion scores, may increase youth's enjoyment and subsequent engagement with them. This is particularly important given that most prevention behaviors are meant to be enacted or assessed routinely [e.g., HIV testing is recommended at least annually and PrEP indications likely to change based on "seasons of risk" (36)]. Within the LYNX app, users are awarded badges such as the Golden Butt (an award for $100 \%$ condom use as a bottom for a month) for recording their sexual behaviors over time.

\section{Delivery of multi-component interventions}

Agent-based modeling suggests that reducing HIV incidence in MSM will require the uptake of multiple prevention services concurrently-for example, HIV testing, condom use, and PrEP and addressing co-morbid conditions (e.g., substance use, mental health issues) that 
disproportionately impact uptake and adherence to these services among YMSM. mHealth interventions are uniquely positioned to deliver multi-component interventions and are thus uniquely positioned to address the syndemic conditions placing YMSM, particularly those that identify as racial or ethnic minority at greater vulnerability. The HealthMindr PrEP app, which has been adapted for youth as MyChoices, is primarily focused on support PrEP uptake, but also screens for potentially problematic substance use [substance use has been associated with delayed PrEP start and lack of PrEP persistence (37)] and refers men to available substance use treatment resources if appropriate.

\section{Will mHealth tools reach the people who need them for HIV prevention?}

Early in the HIV epidemic, there was concern that some communities most impacted by the HIV epidemic - for example, racial/ethnic minorities and people with lower incomes-might not have ready access to mobile phones and adequate bandwidth to support engagement with rich media and prevention services; this concept was called the "digital divide" (38). However, recent data indicate that ownership of smartphones and access to high-speed data services is high among Americans overall, and is highest among young adults [96\% of $18-29$ years old have a smartphone (39)]. Ownership of smartphones is similarly high among white (82\%), Black (80\%) and Hispanic (79\%) Americans (39). There are still some concerns about both levels of cell phone ownership in rural areas, and that the wireless data speed in rural areas is often slower than in urban areas (40). This may be especially problematic for mHealth tools that require higher data throughput speed to provide key services in rural areas, where specialized services are not available. For example, an mHealth approach to remote PrEP delivery for YMSM-ePrEP-utilizes videos and video-based counseling as part of remote PrEP provision (9). Rural areas are a major focus of this research, because of the limited availability of PrEP in many parts of the rural South and thus, rigorously assessing the technologic feasibility of this approach through both quantitative and qualitative evaluations is planned (12). Preliminary experience with the ePrEP intervention indicates that providing an option for phone calls instead of video calls may mitigate the need for high bandwidth, and that most younger users have internet access and are able to access video features (personal communication-Aaron Siegler).

\section{Are public health systems and workforces ready for mHealth?}

The primary responsibility for ensuring the public health lies with governmental agencies at the local, state and federal levels. Health departments are often service providers of last resort for key health services, especially communicable disease control services. When effective mHealth tools for HIV prevention and care are identified and ready for deployment, they might be delivered and managed either by health departments, by community-based organizations who receive direct or indirect funding from the Centers for Disease Control and Prevention (CDC), or by managed care organizations who wish to promote better outcomes for the people in their care. As reviewed elsewhere in this issue, many mHealth apps also include mailout services for HIV and/or STI testing, in which users collect their own specimens and return them to laboratories for analysis. In all phases of these operations-supporting clients to install and use apps, supporting communications with app users through participant management portals, conducting laboratory tests on self-collected samples and alternative specimen types-it is likely that additional training and, in some cases, equipment may be required to prepare and equip public health workforces to provide services through mHealth apps.

Strategies to mitigate these potential barriers to implementation are described in this issue by Paul et al. As part of formative processes in the development of mHealth tools, it is critical to consult with potential end-users to understand early on the training and skills of staff who will be implementing mHealth interventions with clients. A detailed example of a community-driven process for app development has been reported for the HealthMindr app, for which extensive pre-development consultations were held with potential users, potential funders, and potential implementers (41).

\section{Conclusions}

mHealth tools for HIV prevention have proliferated in the past several years, and there is a robust pipeline of new interventions along the HIV care and treatment continua that will yield efficacy results in the coming years. mHealth tools will not replace in-person clinical services and regular engagement with health care for people at risk for or living with HIV or regular engagement with prevention services in community settings But they will likely provide additional 
tools that can help support people with HIV prevention or care needs between clinical visits, enhance clinical care and support, and provide different modes of accessing information, services and prevention commodities. The value and possibilities for mHealth tools can only be fully realized by understanding the things that mHealth tools can do uniquely well, their limitations, and how they fit in the context of the delivery of HIV prevention services in communities.

\section{Acknowledgments}

Funding: This manuscript was completed with support from grant NICHD (U19HD089881) and was facilitated by the Emory University Center for AIDS Research (P30AI050409) and the UNC Center for AIDS Research (P30 AI050410).

\section{Footnote}

Provenance and Peer Review: This article was commissioned by the Guest Editor (Lisa Hightow-Weidman) for the series "Technology-based Interventions in HIV Prevention and Care Continuum among American Youth", published in mHealth. The article has undergone external peer review.

Conflicts of Interest: Both authors have completed the ICMJE uniform disclosure form (available at http://dx.doi. org/10.21037/mhealth-20-71). The series "Technologybased Interventions in HIV Prevention and Care Continuum among American Youth" was commissioned by the editorial office without any funding or sponsorship. LHW served as the unpaid Guest Editor of the series. PSS reports grants from NIH, during the conduct of the study; grants and personal fees from NIH, CDC and Gilead Sciences, personal fees from Merck and Ontario HIV Research Network, outside the submitted work; LHW reports grants from NICHD, during the conduct of the study. Both authors have no other conflicts of interest to declare.

Ethical Statement: The authors are accountable for all aspects of the work in ensuring that questions related to the accuracy or integrity of any part of the work are appropriately investigated and resolved.

Open Access Statement: This is an Open Access article distributed in accordance with the Creative Commons
Attribution-NonCommercial-NoDerivs 4.0 International License (CC BY-NC-ND 4.0), which permits the noncommercial replication and distribution of the article with the strict proviso that no changes or edits are made and the original work is properly cited (including links to both the formal publication through the relevant DOI and the license). See: https://creativecommons.org/licenses/by-nc-nd/4.0/.

\section{References}

1. Sullivan PS, Jones J, Kishore N, et al. The Roles of Technology in Primary HIV Prevention for Men Who Have Sex with Men. Curr HIV/AIDS Rep 2015;12:481-8.

2. Muessig KE, Nekkanti M, Bauermeister J, et al. A systematic review of recent smartphone, Internet and Web 2.0 interventions to address the HIV continuum of care. Curr HIV/AIDS Rep 2015;12:173-90.

3. Maloney KM, Bratcher A, Wilkerson R, et al. Electronic and other new media technology interventions for HIV care and prevention: a systematic review. J Int AIDS Soc 2020;23:e25439.

4. LeGrand S, Knudtson K, Benkeser D, et al. Testing the Efficacy of a Social Networking Gamification App to Improve Pre-Exposure Prophylaxis Adherence (P3: Prepared, Protected, emPowered): Protocol for a Randomized Controlled Trial. JMIR Res Protoc 2018;7:e10448.

5. Sullivan PS, Zahn RJ, Wiatrek S, et al. HIV Prevention Via Mobile Messaging for Men Who Have Sex With Men (M-Cubed): Protocol for a Randomized Controlled Trial. JMIR Res Protoc 2019;8:e16439.

6. Biello KB, Marrow E, Mimiaga MJ, et al. A mobile-based app (MyChoices) to increase uptake of HIV testing and pre-exposure prophylaxis by young men who have sex with men: protocol for a pilot randomized controlled trial. JMIR Res Protoc 2019;8:e10694.

7. Liu A, Coleman K, Bojan K, et al. Developing a Mobile App (LYNX) to Support Linkage to HIV/Sexually Transmitted Infection Testing and Pre-Exposure Prophylaxis for Young Men Who Have Sex With Men: Protocol for a Randomized Controlled Trial. JMIR Res Protoc 2019;8:e10659.

8. Bauermeister J, Sullivan PS, Gravens L, et al. Reducing HIV Vulnerability Through a Multilevel Life Skills Intervention for Adolescent Men (The iREACH Project): Protocol for a Randomized Controlled Trial. JMIR Res Protoc 2018;7:e10174.

9. Siegler AJ, Brock JB, Hurt CB, et al. Advancing PrEP 
Delivery: A Randomized Controlled Trial for Use of ePrEP to Expand PrEP Access to Non-Urban Young Black MSM. JMIR Res Protoc 02/05/2019:13982 (forthcoming/ in press). Available online: http://dx.doi.org/10.2196/ preprints. 13982

10. Sullivan PS, Siegler AJ. Getting pre-exposure prophylaxis (PrEP) to the people: opportunities, challenges and emerging models of PrEP implementation. Sex Health 2018;15:522-7.

11. Siegler AJ, Mayer KH, Liu AY, et al. Developing and Assessing the Feasibility of a Home-based Preexposure Prophylaxis Monitoring and Support Program. Clin Infect Dis 2019;68:501-4.

12. Weiss KM, Bratcher A, Sullivan PS, et al. Geographic Access to PrEP Clinics among Men Who Have Sex with Men in the United States. CROI: Conference on Retroviruses and Opportunistic Infections. Documenting PrEP Deserts. Boston, MA; 2018.

13. Vaughan AS, Kramer MR, Cooper HL, et al. Activity spaces of men who have sex with men: An initial exploration of geographic variation in locations of routine, potential sexual risk, and prevention behaviors. Soc Sci Med 2017;175:1-10.

14. McKenney J, Sullivan PS, Bowles KE, et al. HIV Risk Behaviors and Utilization of Prevention Services, Urban and Rural Men Who Have Sex with Men in the United States: Results from a National Online Survey. AIDS Behav 2018;22:2127-36.

15. Catalani C, Philbrick W, Fraser H, et al. mHealth for HIV Treatment \& Prevention: A Systematic Review of the Literature. Open AIDS J 2013;7:17-41.

16. Stephenson R, Freeland R, Sullivan SP, et al. Home-Based HIV Testing and Counseling for Male Couples (Project Nexus): A Protocol for a Randomized Controlled Trial. JMIR Res Protoc 2017;6:e101.

17. Stephenson R, Metheny N, Sharma A, et al. Providing Home-Based HIV Testing and Counseling for Transgender Youth (Project Moxie): Protocol for a Pilot Randomized Controlled Trial. JMIR Res Protoc 2017;6:e237.

18. MacGowan RJ, Chavez PR, Borkowf CB, et al. Effect of Internet-Distributed HIV Self-tests on HIV Diagnosis and Behavioral Outcomes in Men Who Have Sex With Men: A Randomized Clinical Trial. JAMA Intern Med 2020;180:117-25.

19. Hirshfield S, Downing MJ Jr, Chiasson MA, et al. Evaluation of Sex Positive! A Video eHealth Intervention for Men Living with HIV. AIDS Behav 2019;23:3103-18.

20. 2019 Speedtest U.S. Mobile Performance Report by Ookla
I Speedtest Market Reports [Internet]. [cited 2020 Feb 19]. Available online: https://www.speedtest.net/reports/ united-states/

21. Tuong W, Larsen ER, Armstrong AW. Videos to influence: a systematic review of effectiveness of video-based education in modifying health behaviors. J Behav Med 2014;37:218-33.

22. Hall EW, Sanchez TH, Stein AD, et al. Use of Videos Improves Informed Consent Comprehension in WebBased Surveys Among Internet-Using Men Who Have Sex With Men: A Randomized Controlled Trial. J Med Internet Res 2017;19:e64.

23. Bauermeister JA, Pingel ES, Jadwin-Cakmak L, et al. Acceptability and preliminary efficacy of a tailored online HIV/STI testing intervention for young men who have sex with men: the Get Connected! program. AIDS Behav 2015;19:1860-74.

24. Bauermeister JA, Golinkoff JM, Horvath KJ, et al. A Multilevel Tailored Web App-Based Intervention for Linking Young Men Who Have Sex With Men to Quality Care (Get Connected): Protocol for a Randomized Controlled Trial. JMIR Res Protoc 2018;7:e10444.

25. Sullivan PS, Driggers R, Stekler JD, et al. Usability and Acceptability of a Mobile Comprehensive HIV Prevention App for Men Who Have Sex With Men: A Pilot Study. JMIR Mhealth Uhealth 2017;5:e26.

26. Public Health -- Seattle and King County. FindYourFrequency.com website [Internet]. [cited 2020 May 24]. Available online: http://www.findyourfrequency.com/

27. Horvath KJ, MacLehose RF, Martinka A, et al. Connecting Youth and Young Adults to Optimize Antiretroviral Therapy Adherence (YouTHrive): Protocol for a Randomized Controlled Trial. JMIR Res Protoc 2019;8:e11502.

28. Thakkar J, Kurup R, Laba TL, et al. Mobile Telephone Text Messaging for Medication Adherence in Chronic Disease: A Meta-analysis. JAMA Intern Med 2016;176:340-9.

29. Wise J, Operario D. Use of electronic reminder devices to improve adherence to antiretroviral therapy: a systematic review. AIDS Patient Care STDS 2008;22:495-504.

30. Wald DS, Butt S, Bestwick JP. One-way versus two-way text messaging on improving medication adherence: meta-analysis of randomized trials. Am J Med 2015;128:1139.e1-5.

31. Dasgupta S, Kramer MR, Rosenberg ES, et al. The Effect of Commuting Patterns on HIV Care Attendance Among Men Who Have Sex With Men (MSM) in Atlanta, Georgia. JMIR Public Health Surveill 2015;1:e10. 
32. Siegler AJ, Wirtz S, Weber S, et al. Developing a WebBased Geolocated Directory of HIV Pre-Exposure Prophylaxis-Providing Clinics: The PrEP Locator Protocol and Operating Procedures. JMIR Public Health Surveill 2017;3:e58.

33. Bauermeister JA, Golinkoff JM, Lin WY, et al. Testing the Testers: Are Young Men Who Have Sex With Men Receiving Adequate HIV Testing and Counseling Services?. J Acquir Immune Defic Syndr 2019;82 Suppl 2:S133-S141.

34. Centers for Disease Control and Prevention. Preexposure prophylaxis for the prevention of HIV infection in the United States--2017 update: a clinical practice guideline. Atlanta: US Department of Health \& Human Services, 2018.

35. McDougal SJ, Alexander J, Dhanireddy S, et al. Nonoccupational post-exposure prophylaxis for HIV: 10-year retrospective analysis in Seattle, Washington. PLoS One 2014;9:e105030.

36. Elsesser SA, Oldenburg CE, Biello KB, et al. Seasons of risk: anticipated behavior on vacation and interest in

doi: $10.21037 /$ mhealth-20-71

Cite this article as: Sullivan PS, Hightow-Weidman L. Mobile apps for HIV prevention: how do they contribute to our epidemic response for adolescents and young adults? mHealth 2021;7:36. episodic antiretroviral pre-exposure prophylaxis (PrEP) among a large national sample of U.S. men who have sex with men (MSM). AIDS Behav 2016;20:1400-7.

37. Serota DP, Rosenberg ES, Sullivan PS, et al. Pre-exposure prophylaxis uptake and discontinuation among young black men who have sex with men in Atlanta, Georgia: A prospective cohort study. Clin Infect Dis 2020;71:574-82.

38. Chesser A, Burke A, Reyes J, et al. Navigating the digital divide: a systematic review of eHealth literacy in underserved populations in the United States. Inform Health Soc Care 2016;41:1-19.

39. Center PR. Demographics of mobile device ownership and adoption in the United States. 2018. Available online: https://www.pewresearch.org/internet/fact-sheet/mobile/

40. Coase RH. The federal communications commission. In: Private and Common Property. J Law Econ 1959;2:1-40

41. Goldenberg T, McDougal SJ, Sullivan PS, et al. Building a Mobile HIV Prevention App for Men Who Have Sex With Men: An Iterative and Community-Driven Process. JMIR Public Health Surveill 2015;1:e18. 